\title{
SIMULTANEOUS BILATERAL TRABECULECTOMY
}

\author{
C. E. HUGKULSTONE, L. STEVENSON and S. A. VERNON \\ Nottingham
}

\begin{abstract}
SUMMARY
In a retrospective study, 24 patients who had undergone simultaneous bilateral trabeculectomy over a 6-year period were reviewed. The duration of disease was at least 2 years, indicating that surgery was not performed as a primary procedure. No patients suffered complications leading to bilateral blindness, although $6(25 \%)$ patients had reduced vision in both eyes at the first postoperative visit, with $3(13 \%)$ worse than $6 / 36$ binocularly. There was no evidence of an asymmetric response in the fall in intraocular pressure following trabeculectomy, after a mean follow-up of over 3 years. Compared with a matched group of patients undergoing unilateral trabeculectomy, the simultaneous bilateral group had a similar period of hospital stay. However, this was no shorter than that found in a group having staged bilateral surgery during the same admission, over the same study period. When separate admissions were required for bilateral drainage operations, though, there was a significant increase in the total length of inpatient stay. No convincing advantage was found for simultaneous bilateral trabeculectomy.
\end{abstract}

The concept of simultaneous bilateral intraocular surgery is anathema to many clinicians because of the fear of bilateral complications. Simultaneous bilateral cataract surgery was first suggested nearly 40 years ago ${ }^{1}$ and interest has been rekindled recently. ${ }^{2-5}$ Advantages claimed for this technique include a shorter hospital stay and reduced costs compared with separate operations,,$^{2.3 .6-9}$ a single general anaesthetic, ${ }^{2-4.6 .7}$ greater patient satisfaction ${ }^{7.8}$ with quicker binocular visual rehabilitation ${ }^{1.7}$ and reduced waiting lists. ${ }^{7}$

However, a long delay in performing surgery to the second eye does not prejudice the results of cataract extraction. This may not be so with drainage surgery, and perhaps glaucoma presents a stronger case for simultaneous bilateral surgery. Although Fenton and Gardner ${ }^{7}$ briefly mentioned that bilateral drainage surgery was performed in their Unit, they did not analyse the results any

Correspondence to: Mr C. E. Hugkulstone, FRCSEd, FRCOphth, Academic Unit of Ophthalmology, University Hospital, Nottingham NG7 2UH, UK. further. We report here the results of the first study of simultaneous bilateral trabeculectomy.

\section{METHODS}

All patients who had undergone trabeculectomy between January 1985 and December 1990 were identified from theatre records, and the case-notes of those who had had simultaneous bilateral trabeculectomy were reviewed (group A).

The following data were recorded: the sex of the patient and age at the time of surgery, the glaucoma diagnosis and the duration of disease before admission. The intraocular pressure (IOP) was noted for each eye at diagnosis, on admission, at the first post-operative visit and at 6 months, 1 year and at the final visit, as were the number of ocular hypotensive medications. On admission, the visual acuity for each eye was recorded, with spectacles when appropriate. Anaesthetic technique and the grade of surgeon for each eye were identified, as were post-operative complications. The length of hospital stay was also noted.

At the first post-operative visit, visual acuities were again recorded, with spectacles if worn but without further correction with a pinhole. Any late complications were noted. Follow-up was defined as the time from surgery until the final visit recorded in the case-notes.

To compare the immediate post-operative complication rate, duration of hospital stay and the effects on visual acuity, three further groups of patients were also reviewed. The first included patients who had undergone unilateral trabeculectomy (group B). These were matched to group A, from theatre records, for age and sex, date of surgery, surgical technique and consultant. The second (group C) consisted of all patients, identified from theatre records, who had had staged bilateral drainage surgery during a single hospital admission over the same study period but with less than 3 days between operations (to minimise the effects of a longer delay on the incidence of bilateral complications). The final group (group D) was included to study the effects on the IOP in the unoperated fellow eye of a delay of from 3 to 9 months between drainage operations. These patients were age- and sex-matched to group A, again from theatre records. 
Results are given as the mean (SD). Statistical analysis was performed with paired and unpaired $t$-tests as appropriate. Frequencies were compared using the chi-squared test or Fisher's exact probability test.

\section{RESULTS}

During the study period 822 trabeculectomies were performed, of which $24(2.9 \%)$ were simultaneous bilateral trabeculectomies (group A). There were 8 men and 16 women in this group, with a mean age of 65.6 (14.3) years. Eighteen $(75 \%)$ were diagnosed as having chronic openangle glaucoma and $5(21 \%)$ as having chronic narrowangle glaucoma, whilst the remaining patient $(4 \%)$ had lower tension glaucoma. The mean duration of disease before surgery was 24.1 (27.0) months, and the follow-up was 43.8 (23.8) months. All patients had a minimum follow-up of 6 months, but 2 patients were not seen subsequently.

Group B (unilateral trabeculectomies) contained 8 men and 16 women, with a similar mean age $(66.1$ (15.4) years) to group A. Group C (staged bilateral trabeculectomies) consisted of 18 patients ( 9 men, 9 women) with a mean age of 73.2 (9.4) years; this was not significantly different from groups A and B, although approached significance compared with group D ( 8 men, 16 women; mean age 65.4 (14.2) years; $p=0.051$ ).

In group $\mathrm{A}$, surgery was performed under general anaesthesia in $23(96 \%)$ of the 24 patients, and by the same surgeon in $10(42 \%)$. Group B had surgery under general anaesthesia in 18 cases $(75 \%, p=0.021)$. In group C, local anaesthesia was used for both eyes in $15(83 \%)$ of the 18 patients, and $13(72 \%)$ operations were by the same surgeon. The local anaesthetic was given using a retrobulbar technique which should not affect the outcome of trabeculectomy, unlike the recent report of improved IOP control when the subconjunctival route is employed.$^{10}$ For group D, general anaesthesia was employed for both eyes in $10(42 \%)$ of the 24 patients $(p=0.0002$ compared with group A), and 8 (33\%) had their operations performed by the same surgeon.

Bilateral post-operative complications (hyphaema, shallow/flat anterior chamber or both) occurred in 18 $(75 \%)$ patients in group $\mathrm{A}$, in $9(50 \%)$ in group $\mathrm{C}$ and in 12 $(50 \%)$ in group D. These differences did not achieve statistical significance, and were comparable to the incidence of complications (in one eye) in group B $(15 ; 63 \%)$.

For group A, the mean inpatient stay was 6.3 (3.6) days. This was similar to that in group B (5.3 (2.3) days) and group C (7.2 (2.8) days). If the interval between operations

Table I. Mean (SD) intraocular pressures $(\mathrm{mmHg}$ ) following simultaneous bilateral trabeculectomy (group A)

\begin{tabular}{lll}
\hline & Right eye & Left eye \\
\hline Diagnosis & $37.6(12.7)$ & $35.6(10.4)$ \\
Admission & $26.0(8.1)$ & $25.7(6.1)$ \\
First post-operative & $11.5(5.4)$ & $15.1(11.7)$ \\
6 months & $14.0(6.5)$ & $15.6(14.8)$ \\
1 year & $14.5(6.5)$ & $15.3(11.8)$ \\
Final visit & $14.0(5.1)$ & $13.7 \quad(4.4)$ \\
\hline
\end{tabular}

in this latter group (2.4 (0.5) days) is subtracted from the patients' duration of hospital stay, then group $\mathrm{C}$ has the shortest inpatient stay (4.8 (2.9) days), although this did not achieve statistical significance. However, the total duration of inpatient stay in group D (10.8 (3.3) days) was significantly longer compared with the other two groups who underwent bilateral surgery $(p<0.0001$ versus group $\mathrm{A}$, and $p<0.001$ versus group C).

At the first post-operative visit, 7 (29\%) patients in group A had a reduction in visual acuity of 2 lines or more of Snellen acuity in one eye and a further $6(25 \%)$ in both eyes. These frequencies were lower (although not statistically significant) in group C $(2(11 \%)$ and $3(17 \%)$, respectively). In group B, 8 (33\%) patients had a similar reduction in visual acuity in the operated eye. This incidence was not statistically different from the frequency of a similarly reduced vision in one or both eyes in either of these two groups. In group A, $3(13 \%)$ patients who had a bilateral fall in vision would have been eligible for BD8 registration as partially sighted (vision worse than 6/36, without regard to visual field loss) at the first post-operative visit, compared with $1(6 \%)$ in group C. Only 1 patient $(4 \%)$ in group B was similarly visually handicapped at the first post-operative visit, following a transient reduction of vision in the operated eye (from 6/24 to counting fingers) because the fellow eye had a pre-operative acuity of counting fingers from advanced glaucomatous damage. This situation did not occur in the other two groups. Not surprisingly, no patients in group D lost vision in both eyes following either of their two admissions.

Two (8\%) patients in group A lost vision permanently in one eye (vision in both cases falling from $6 / 18$ to counting fingers). One case occurred immediately post-operatively with loss of residual field, whilst the other was associated with an uncontrollable rise in IOP after 1 week. This eye eventually became phthisical 46 months after drainage surgery. No permanent visual loss occurred in the other three groups.

The mean IOPs following simultaneous bilateral trabeculectomy (group A) are shown in Table I. No significant

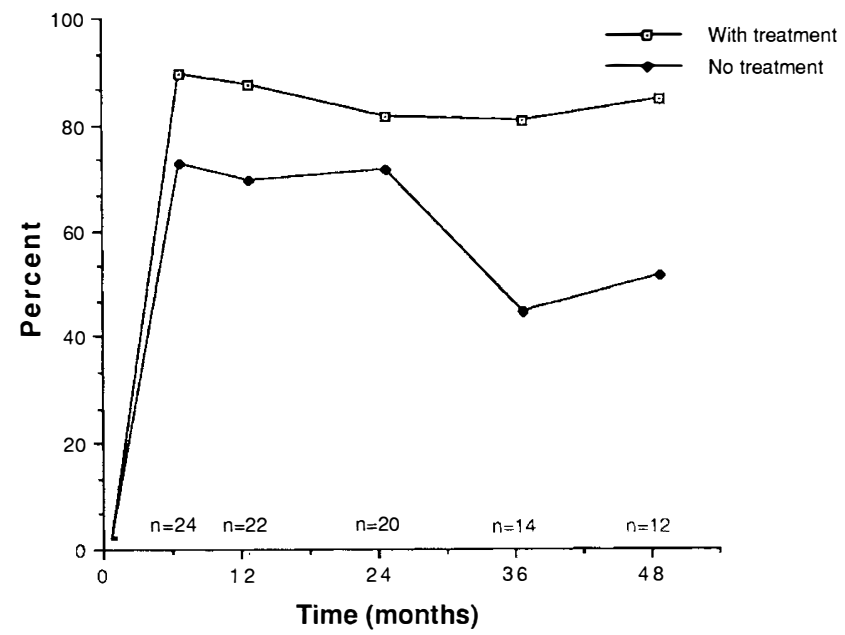

Fig. 1. Proportion of patients with an intraocular pressure $<22 \mathrm{mmHg}$ in both eyes with and without treatment following simultaneous bilateral trabeculectomy (group A). 
Table II. Behaviour of intraocular pressure $(\mathrm{mmHg})$ and number of treatments in the unoperated eye following initial trabeculectomy (group D)

\begin{tabular}{|c|c|c|c|c|}
\hline & \multicolumn{2}{|c|}{$\begin{array}{l}\text { First post-operative vs } \\
\text { first admission }\end{array}$} & \multicolumn{2}{|c|}{$\begin{array}{l}\text { Second admission vs } \\
\text { first admission }\end{array}$} \\
\hline & IOP & Treatment & IOP & Treatment \\
\hline Unchanged & $3(13 \%)$ & $20(83 \%)$ & $3(13 \%)$ & $18(75 \%)$ \\
\hline Increased & $14(58 \%)$ & $1(4 \%)$ & $9(38 \%)$ & $4(17 \%)$ \\
\hline Decreased & $7(29 \%)$ & $3(13 \%)$ & $12(50 \%)$ & $2(8 \%)$ \\
\hline
\end{tabular}

asymmetry was seen in the response to trabeculectomy between right and left eyes. Fig. 1 shows the proportion of patients in this group achieving an IOP $<22 \mathrm{mmHg}$ in both eyes with and without treatment up to 4 years following surgery.

To assess the behaviour of IOP in the unoperated fellow eye in group D, IOP was recorded at both the first postoperative visit following the initial trabeculectomy (after a mean of 13.5 (7.0) days from discharge), and on admission for the subsequent drainage procedure to the fellow eye (after a mean of 4.5 (1.7) months), and was compared with the IOP in that eye on admission for the first trabeculectomy. The numbers of ocular hypotensive medications were compared in a similar manner. These results are given in Table II.

Apart from the case of phthisis mentioned above, 2 (8\%) other patients in group A developed late complications. One had a unilateral bleb infection 23 months postoperatively which led to loss of IOP control. The other patient developed bilateral malignant glaucoma 5 months after surgery. However, IOP control was maintained with mydriatic drops thereafter. One patient in group $\mathrm{C}$ suffered a unilateral bleb leak 19 months post-operatively, but there was no long-term effect on IOP control. No late complications occurred in groups B and D.

\section{DISCUSSION}

The major potential disadvantage of simultaneous bilateral intraocular surgery is the risk of serious peri- or postoperative complications leading to blindness. " Bilateral expulsive haemorrhage has rarely been reported following cataract surgery, although not occurring simultaneously. ${ }^{12}$ Benezra and Chirambo" reported a solitary case of bilateral endophthalmitis following simultaneous cataract extractions, and there is one case of bilateral expulsive haemorrhage occurring after simultaneous iridencleisis without sutures. ${ }^{12}$ Thus these fears do not appear to be supported by the literature, although even a single case will have a devastating effect on that patient. Alternatively, this paucity of reports of bilateral blinding complications following intraocular surgery may simply reflect surgeons' reluctance to record such cases. However, the incidence of expulsive haemorrhage following trabeculectomy has been reported as $1.6 \%$ in a series of 305 consecutive procedures. ${ }^{13}$ Therefore, in our sample of only 24 cases undergoing simultaneous bilateral trabeculectomy, only 0.4 episodes of unilateral expulsive haemorrhage would be expected.
Simultaneous bilateral surgery should offer a saving in portering duties, as there is half the number of visits to theatre. Also, whilst trabeculectomy is often performed under local anaesthesia, if a general anaesthetic is required (at the request of either the surgeon or the patient), then simultaneous surgery has the advantage of a single anaesthetic - as pointed out by advocates of simultaneous cataract extraction. ${ }^{2-4.6 .7}$ This reduces the risks of re-exposure to anaesthetic agents within a short time, and should also improve turnaround time in theatre compared with separate operations. However, as groups A and B were matched for consultant (and thus surgical management), the higher frequency of general anaesthesia in group A suggests that the choice of anaesthetic technique may be influenced by the decision to perform either unilateral or simultaneous bilateral surgery. The higher frequency of local anaesthesia in group $\mathrm{C}$ may reflect surgeon preference, as 11 $(61 \%)$ of this group were under the care of one consultant, and all but 1 of these were operated on under local anaesthetic. Naturally, it is important that patients are recleaned and redraped for each eye, and that the surgeon scrubs between operations when performing simultaneous bilateral trabeculectomy, as previously suggested for cataract surgery. $2,3,5,7$

That the period of hospital stay was similar for both unilateral and simultaneous bilateral trabeculectomies suggests that the latter option may offer a saving in admission time and costs. However, the staged surgery group (group C) did not have a significantly longer time in hospital than the simultaneous bilateral trabeculectomy group (group A): the inpatient stay in group $\mathrm{C}$ was only 1 day longer than in group $\mathrm{A}$, despite approximately 2.5 days between operations. The obvious advantage of staging surgery is that it allows postponement of the second operation should circumstances dictate. However, if the operations require separate admissions (group D), then a significant increase in the total length of inpatient stay (plus associated costs) occurs.

Overall, the incidence of complications in both groups having bilateral trabeculectomies during the same admission (groups $\mathrm{A}$ and $\mathrm{C}$ ) was no greater than in those undergoing unilateral surgery (group B), and not dissimilar to that previously reported for unilateral surgery, ${ }^{14,15}$ although higher than others. ${ }^{16.17}$ However, the bilateral, albeit transient reduction in visual acuity found, particularly following simultaneous bilateral trabeculectomy, may seriously impair the patient's ability to manage at home in the short term. The visual acuities were not best corrected, but this does represent the level of vision that the patient must manage with. Staged surgery, as mentioned above, offers the advantage of postponing the second operation if vision is impaired in the first eye (by hyphaema, for example). The routine use of mydriatics (especially atropine) after trabeculectomy would also militate against bilateral surgery (simultaneous or staged) during the same admission, for similar reasons.

However, separate admissions for bilateral surgery may lead to a rise in IOP in the unoperated eye during the inter- 
val between operations, especially if systemic treatment (with carbonic anhydrase inhibitors) is stopped after the first trabeculectomy. Indeed, 58\% of group D had a rise in IOP in the fellow eye at the first post-operative visit (of 4.9 (4.1) $\mathrm{mmHg}$ ), and $38 \%$ had a rise at the second admission (of $5.4(3.2) \mathrm{mmHg}$ ), compared with the initial admission. This finding is difficult to explain, as the number of ocular hypotensive medications was unchanged in $83 \%$ and $75 \%$ of this group, respectively. The rise in IOP may have a deleterious effect on the visual field in this eye, particularly if advanced glaucomatous damage already exists or if the second operation is delayed for some time.

As this was a retrospective study, it was impossible to identify from the case-notes the rationale for choosing simultaneous bilateral trabeculectomy. However, surgeon preference undoubtedly played a part, as nearly all cases in this group $(19,79 \%)$ were under the care of one consultant. The mean duration of disease before surgery was at least 2 years, indicating that surgery was not chosen as the primary treatment. ${ }^{18}$

In conclusion, no striking advantage was found for performing simultaneous bilateral trabeculectomy, although no patient suffered simultaneous complications leading to bilateral blindness. However, the temporary deleterious effect on bilateral visual acuity found in this study deserves special attention. Because of this, we would not recommend simultaneous bilateral trabeculectomy with current surgical techniques. If both eyes do require surgery urgently then we would advocate staged surgery, perhaps during the same hospital admission. To investigate this further would require a carefully considered and wellcontrolled prospective study.

We are grateful to Messrs N. R. Galloway, D. Knight-Jones, P. M. Jacobs, S. M. Haworth and S. N. Rizk for kindly allowing us to study patients under their care.

Key words: Bilateral simultaneous surgery, Complications, Hospital stay, Trabeculectomy.

\section{REFERENCES}

1. Duthie OM. Bilateral cataract extraction. Trans Ophthalmol Soc UK 1955;75:25-31.
2. Akingbehin T, Sunderraj P. Simultaneous bilateral lens implantation: is the procedure justified? Eur $\mathbf{J}$ Implant Refract Surg 1992;3:131-3.

3. Harfitt R, Moriarty A, Mastellone G. Is simultaneous bilateral cataract extraction with lens implantation justified? Eur J Implant Refract Surg 1992;3:134-7.

4. Adhikary HP, Harrington L. Simultaneous bilateral intraocular lens implantation. Eur J Implant Refract Surg 1992;3:138-9.

5. Nielsen PJ. Simultaneous bilateral cataract extraction: ECCE versus ICCE. Eur J Implant Refract Surg 1992; 3:140-4.

6. Joseph N, David R. Bilateral cataract in one session: report on five years experience. Br J Ophthalmol 1977;61:619-21.

7. Fenton PJ, Gardner ID. Simultaneous bilateral intraocular surgery. Trans Ophthalmol Soc UK 1982;102:298-301.

8. Jardine P. Simultaneous bilateral cataract extraction. Trans Ophthalmol Soc UK 1970;70:719-24.

9. Benezra D, Chirambo MC. Bilateral versus unilateral cataract extraction: advantages and complications. Br J Ophthalmol 1978;62:770-3.

10. Noureddin BN, Jeffrey M, Franks WA, Hitchings RA. Conjunctival changes after subconjunctival lignocaine. Eye 1993; 7:457-60.

11. Shepard DD. Ed. Are there any indications for simultaneous bilateral cataract-intraocular lens (IOL) surgery? J Cataract Refract Surg 1988;14:339-45.

12. Payne JW, Kameen AJ, Jensen AD, Christy NE. Expulsive haemorrhage: its incidence in cataract surgery and a report of four bilateral cases. Trans Am Ophthalmol Soc 1985; 83:181-204.

13. Givens K, Shields MB. Suprachoroidal haemorrhage after glaucoma filtering surgery. Am J Ophthalmol 1987; 103:689-94.

14. Zaidi AA. Trabeculectomy: a review and 4-year follow-up. Br J Ophthalmol 1980;64:436-9.

15. Watson PG, Jakeman C, Ozturk M, Barnett MF, Barnett F, Khaw KT. The complications of trabeculectomy: a 20-year follow-up. Eye 1990;4:425-38.

16. Watson PG, Barnett F. Effectiveness of trabeculectomy in glaucoma. Am J Ophthalmol 1975;79:831-45.

17. D'Ermo F, Bonomi L, Doro D. A critical analysis of the long-term results of trabeculectomy. Am J Ophthalmol 1979;88:829-35.

18. Jay JL, Murray SB. Early trabeculectomy versus conventional management in primary open angle glaucoma. $\mathrm{Br} \mathrm{J}$ Ophthalmol 1988;72:881-9. 